DOI: 10.20472/EFC.2018.010.037

\author{
KAROLINA TURA-GAWRON \\ Gdansk University of Technology , Poland
}

MAGDALENA SZYSZKO

WSB University in Poznan, Poland

\title{
SPATIAL APPROACH TO HETEROGENEITY OF INFLATION EXPECTATIONS IN THE EURO AREA
}

\begin{abstract}
:
In this article, we examine the spatial heterogeneities in inflation expectations of the euro area consumers. We expect to find them heterogeneous in our research period of 2001-2016. Contrary to standard examination of heterogeneity, a spatial correlation analysis is applied by referring to global and local correlation measures. It is performed with the economic distance-based weights (the difference in HICP rates). Application of spatial analysis is the main contribution of our examination. Standard examinations ignore spatial relations and might be misleading. Our findings suggest that expectations are heterogeneous once the differences of inflation rates represent economic distance between the countries that we cover by our examination.
\end{abstract}

\section{Keywords:}

inflation expectations, expectations heterogeneity, euro area, spatial analysis

JEL Classification: E52, E61, C31 


\section{Introduction}

This study covers consumer inflation expectations in the Member States of the euro area. The research hypothesis assumes that inflation expectations across the euro area Member States are not homogenous, even though some coordination should exist due to the European Central Bank's (ECB) common monetary policy, an area-wide goal of price stability and common monetary and financial indicators, such as money supply, interbank rates, exchange rate, or even common inflation projections. The national factors might prevail while affecting the formation of expectations. Our assumption, expressed in terms of spatial analysis, is as follows: we do not expect that strong and stable general spatial correlations of expectations and clusters exist. Thus, we expect to support the research that highlights national, rather than European, factors in the formation of expectations: there is no general tendency to mimic some leading countries (such as Germany).

In this paper, we analyse heterogeneity by means of spatial analysis which we find the most important contribution of our paper. According to Tobler (1970), the first law of geography is that everything is related to everything else, but near things are more related than are the distant things. Although spatial analysis originated from geography or social geography, it finds more common application in economics, where the neighbourhood is expressed not by the distance in space but by the difference in economic performance. Then, we verify whether expectations are shaped by their close spatial and economic structure (spatial clustering). To the best of our knowledge, there has been no research conducted on spatial dependence between expectations. We conduct a spatial correlation analysis of the expectations by referring to global and local correlations' measures. With the application of the spatial approach we avoid the criticism of Anselin (1988); i.e., that ignoring spatial dependence may be misleading.

In the following research, we consider eighteen euro area Member States. Lithuania as the most recent joiner is excluded from the sample. The coverage of the sample varies accordingly to new euro area enlargement: the new euro area Member States are included in the sample according to the year of their euro adoption. The time span covers 2002-2016.

\section{Literature overview}

There are several studies that analyse the heterogeneity of expectations across the Member States of the euro area. They generally suggest that expectations are not homogenous and they supported our first hypothesis. Quite recent example by Gimeno \& Ortega (2016) explored the behaviour of inflation expectations in France, Germany, Italy, and Spain. They pinpointed considerable differences in inflation expectations across these countries only at short maturities. Similar results were provided earlier by Gnan, Langthaler, \& Valderrama (2011) for Austria, France, Finland, Germany, Italy, Ireland, Netherlands, Portugal, Slovakia, Slovenia, and Spain. Cross-country heterogeneity is 
also captured while the learning rule of the formation of expectations is examined (Weber, 2009), (Strobach \& Cruisen, 2015).

Some geographical implications for the formation of expectations are presented in the papers of Berger, Ehrmann, \& Fratzscher (2009). They analysed whether the ability of professional forecasters to understand and to anticipate the ECB's monetary policy depends on economic and non-economic factors, including their location in relation to the central bank's headquarters. They explored the notable differences in forecast accuracy and showed that they are partly related to geography and clustering around information hubs, as well as to country-specific economic conditions. The same authors drew similar conclusions on the geographic importance of forecasters for the US (Berger, Ehrmann \& Fratzscher, 2011). Their papers presented the geographic context for the formation of expectations by professionals but they did not apply the spatial approach presented in our research. In both research papers, an individual dataset was used instead of aggregate expectations of the group of forecasters. Although our research search for spatial deployment of expectations, this is not the kind of geographic deployment that we are looking for in this study: the authors apply traditional methodology but they introduce variables that represents geographic distance. Spatial analysis relates the relation to the distance between countries. In our case we refer more to economic distance.

\section{Sample and methodology}

The consumers' inflation expectations are obtained from the Business and Consumers Surveys conducted monthly by the European Commission. We quantify them using the probability method based on Carlson \& Parkin (1975) and Batchelor \& Orr (1988). The monthly data on the HICP inflation rate were downloaded from the Eurostat database. The map shapes come from a GIS database.

Our methodology consists of spatial analysis of the inflation expectations in our sample. To test whether inflation expectations in each country are spatially correlated over consecutive years, we use a Univariate (Global) Moran's I test, which is a weighted autocorrelation coefficient used to detect departures from spatial randomness (Anselin, Florax, \& Rey, 2004). Moran's I statistic is provided by Cliff \& Ord (1981):

$$
I=\frac{n \sum_{i=1}^{n} \sum_{j=1}^{n} w_{i j}\left(x_{i}-\bar{x}\right)^{2}\left(x_{j}-\bar{x}\right)^{2}}{\sum_{i=1, i \neq j}^{n} \sum_{j=1}^{n} w_{i j} \sum_{i=1}^{n}\left(x_{i}-\bar{x}\right)^{2}}
$$

where: $n$ - number of spatial units, $x_{i}$-variable under consideration in region $i, x_{j}-$ variable under consideration in region $j, \bar{x}$-average value of the variable across all regions, and $w_{i j}$-generic element (at location $i$, $)$ of an $n \times n$ positive and non-stochastic 
matrix of weights $W \equiv\left\{w_{i j}\right\}$. The values of $w_{i j}$ depend on the assumed definition of the spatial relations among locations.

Next, the Univariate Local Moran's I coefficients are calculated. Univariate Local Moran's I is one of the Local Indicators of Spatial Association (LISA), and it is expressed by the following formula (Anselin, 1995):

$I_{i}=\left(x_{i}-\bar{x}\right) \sum_{j=1}^{n} w_{i j}\left(x_{j}-\bar{x}\right)$

- notation is as in Eq. (1). Its value for each observation (each Member State) indicates the extent of significant spatial clustering of similar values around that observation, and the sum of LISAs for all observations is proportional to the global indicator of spatial association (Anselin, 1995). With Univariate Local Moran's I we detect local spatial autocorrelation: the association between the value observed at a location and the values observed at the sites nearby. We also determine local clusters (regions where adjacent areas have similar values) and outliers (areas distinct from their neighbours), the so called hot-spots (Anselin, Florax, \& Rey, 2004).

First, global autocorrelation is measured to check the global correlation among the inflation expectations in our sample in consecutive years. To establish the matrix of economic distance weights we use inflation rates registered nationally for each Member State that we cover. The literature review on inflation expectations drivers did not deliver a better option for economic distance specification: inflation is the most important driver of inflation expectations. It is the common point of quite diverse examinations on expectations, see i.a.: Cerisola \& Gelos (2009), Ciccarelli \& Garcia (2009), Gambetti \& Moretti (2016). The economic distance is calculated with the use of Euclidean distance $d_{i j}$ between the variable chosen for a weight in countries $i$ and $j$ (Cliff \& Ord, 1981). To calculate economic distance weights, we apply the threshold distance weights. This assumes that euro area Member State $i$ is a neighbour of Member State $j$ if the distance between them is less than a specified maximum distance:

$w_{i j}= \begin{cases}1, & 0 \leq d_{i j} \leq d_{\max } \\ 0, & d_{i j}>d_{\max } .\end{cases}$

The economic distance is measured by a Euclidean metric $d_{i j}$. In this case, each Eurozone member state has at least one neighbour.

The positive Univariate Global Moran's I coefficient value indicates similarity across the countries (either high or low), the negative value indicates dissimilarity, and the value close to zero indicates randomness of deployment (Anselin, Florax, \& Rey, 2004). 
The global and local autocorrelations investigate the heterogeneity of inflation expectations across the euro area Member States over consecutive years. In the cluster analysis, hot-spots were recognised that enabled the states to be grouped into our categories: High-High, Low-Low, Low-High, High-Low. The High-High (Low-Low) case stands for high (low) inflation expectations in one Member State and its neighbours. Such cases represent clusters. The High-Low (Low-High) case stands for high (low) inflation expectations in one state and low (high) inflation expectations in its neighbours. Such cases are classified as outliers.

To investigate the significance of the Global Moran's I coefficients we perform a Monte Carlo randomisation test in which the null hypothesis assumes the spatial randomness of the time series. We apply a pseudo $p$ value derived from a conditional random permutation procedure that generates the reference distribution using 999 permutations (Anselin, 2005). For Local Moran's coefficients the significance was tested by comparison with a reference distribution obtained by 999 permutations (Anselin, 1995).

We are aware that the statistics of spatial dependence are large sample tests. The number of countries we have covered in our sample ranges from eleven to eighteen, which is rather small for a standard spatial analysis. However, Anselin \& Florax (1995) tested Moran's I statistics for a finite sample and confirmed their usefulness.

\section{Results}

The starting point for the analysis of economic deployment is the establishment of the economic distance weight system. For each observation we use two months' lagged HICP rates registered in each Member State, respectively. The Global Univariate Moran's I statistics are statistically significant for most of the cases, indicating some positive spatial correlation of inflation expectations across the countries that share similar inflation rates (Tab. 1). This means that spatial deployment of inflation expectations is, to some extent, economical.

Table 1. Univariate Global Moran's I test results for economic deployment

\begin{tabular}{|c|c|c|c|c|c|}
\hline Year & $\begin{array}{c}\text { Global Univariate } \\
\text { Moran's I } \\
\text { Coefficient }\end{array}$ & $\begin{array}{l}\text { Pseudo } \\
\text { p-value }\end{array}$ & Year & $\begin{array}{c}\text { Global Univariate } \\
\text { Moran's I } \\
\text { Coefficient }\end{array}$ & Pseudo p-value \\
\hline $\begin{array}{l} \\
2002\end{array}$ & $0.69^{\star \star}$ & 0.004 & V 2009 & -0.06 & 0.34 \\
\hline $\begin{array}{l}X I \\
2002\end{array}$ & $0.86^{\star \star \star}$ & 0.001 & $\begin{array}{l}X I \\
2009\end{array}$ & -0.12 & 0.24 \\
\hline $\begin{array}{l}V \\
2003\end{array}$ & $0.32^{*}$ & 0.017 & V 2010 & -0.3 & 0.14 \\
\hline $\begin{array}{l}X \mathrm{XI} \\
2003\end{array}$ & $0.77^{\star \star}$ & 0.002 & $\begin{array}{l}X I \\
2010\end{array}$ & $0.22^{\star \star}$ & 0.003 \\
\hline $\begin{array}{l}V \\
2004\end{array}$ & $0.012^{*}$ & 0.014 & V 2011 & 0.08 & 0.12 \\
\hline $\begin{array}{l}X I \\
2004\end{array}$ & $0.28^{\star *}$ & 0.006 & $\begin{array}{l}X \mathrm{XI} \\
2011\end{array}$ & $0.07^{*}$ & 0.09 \\
\hline V & $0.46^{* *}$ & 0.003 & V 2012 & 0.05 & 0.19 \\
\hline
\end{tabular}




\begin{tabular}{|c|c|c|c|c|c|}
\hline 2005 & & & & & \\
\hline $\begin{array}{l}X I \\
2005\end{array}$ & $0.2^{*}$ & 0.09 & $\begin{array}{l}X I \\
2012\end{array}$ & $0.06^{*}$ & 0.035 \\
\hline $\begin{array}{l}V \\
2006\end{array}$ & $0.57^{*}$ & 0.012 & V 2013 & $0.43^{\star *}$ & 0.003 \\
\hline $\begin{array}{l}X I \\
2006 \\
\end{array}$ & $0.76^{* *}$ & 0.002 & $\begin{array}{l}X I \\
2013 \\
\end{array}$ & $0.61^{* * *}$ & 0.001 \\
\hline $\begin{array}{l}V \\
2007\end{array}$ & $0.53^{\star \star *}$ & 0.001 & V 2014 & $0.14^{\star}$ & 0.1 \\
\hline $\begin{array}{l}X I \\
2007\end{array}$ & $0.71^{\text {*** }}$ & 0.001 & $\begin{array}{l}X I \\
2014\end{array}$ & $0.1^{*}$ & 0.06 \\
\hline $\begin{array}{l}V \\
2008\end{array}$ & $0.009^{*}$ & 0.1 & V 2015 & 0.05 & 0.14 \\
\hline $\begin{array}{l}X I \\
2008 \\
\end{array}$ & -0.14 & 0.2 & $\begin{array}{l}\text { XI } \\
2015 \\
\end{array}$ & -0.07 & 0.5 \\
\hline $\begin{array}{l}V \\
2009 \\
\end{array}$ & -0.06 & 0.34 & V 2016 & -0.065 & 0.5 \\
\hline $\begin{array}{l}X I \\
2009\end{array}$ & -0.12 & 0.24 & $\begin{array}{l}X I \\
2016 \\
\end{array}$ & $0.21^{*}$ & 0.09 \\
\hline
\end{tabular}

Table 1. Univariate Global Moran's I test results for economic deployment

Significant at ${ }^{*} p<0.1,{ }^{* *} p<0.01,{ }^{* * *} p<0.001$

Note: the sample changes due to the new Member States' accession to the euro area.

Source: own calculations.

The Local Moran's I autocorrelation Test (Tab. 2) shows clusters and outliers that exist for economic deployment. This means that there are Member States that group around other Member States with similar (clusters) or different (outliers) inflation rates. If Spain is such a Member State, a cluster (outlier) means that the inflation expectations in Spain and other countries with a similar inflation rate at a particular moment correlate positively (negatively).

Table 2. Univariate Local Moran's I test results for economic deployment

\begin{tabular}{|c|c|c|c|c|c|}
\hline Year & $\begin{array}{c}\text { Euro area } \\
\text { member } \\
\text { states }\end{array}$ & Autocorrelation & Cluster map & $\begin{array}{l}\text { Pseudo } \\
\text { p-value }\end{array}$ & $\begin{array}{l}\text { Interpretation } \\
\text { (Cluster/outlier) }\end{array}$ \\
\hline \multirow{7}{*}{ V 2002} & Italy & Positive & 'Low-Low' & 0.05 & Cluster \\
\hline & Germany & Positive & 'Low-Low' & 0.001 & Cluster \\
\hline & Belqium & Positive & 'Low-Low' & 0.05 & Cluster \\
\hline & Spain & Positive & 'Low-Low' & 0.05 & Cluster \\
\hline & France & Positive & 'Low-Low' & 0.001 & Cluster \\
\hline & Netherlands & Positive & 'High-High & 0.05 & Cluster \\
\hline & \begin{tabular}{|l|} 
Finland \\
\end{tabular} & Positive & 'Low-Low' & 0.05 & Cluster \\
\hline \multirow{8}{*}{ XI 2002} & Portugal & Positive & 'High-High & 0.001 & Cluster \\
\hline & Greece & Positive & 'High-High & 0.01 & Cluster \\
\hline & Netherlands & Positive & 'High-High & 0.001 & Cluster \\
\hline & France & Positive & 'Low-Low' & 0.05 & Cluster \\
\hline & Germany & Positive & 'Low-Low' & 0.05 & Cluster \\
\hline & Belgium & Positive & 'Low-Low' & 0.05 & Cluster \\
\hline & Finland & Positive & 'Low-Low' & 0.001 & Cluster \\
\hline & Austria & Positive & 'Low-Low' & 0.001 & Cluster \\
\hline V 2003 & \begin{tabular}{|l|} 
Portugal \\
\end{tabular} & Positive & 'High-High' & 0.05 & Cluster \\
\hline
\end{tabular}




\begin{tabular}{|c|c|c|c|c|c|}
\hline & France & Positive & 'Low-Low' & 0.05 & Cluster \\
\hline & Germany & Positive & 'Low-Low' & 0.05 & Cluster \\
\hline & Italy & Negative & 'Low-High’ & 0.05 & Outlier \\
\hline \multirow{7}{*}{ XI 2003} & Finland & Positive & ‘Low-Low' & 0.01 & Cluster \\
\hline & Germany & Positive & 'Low-Low' & 0.05 & Cluster \\
\hline & Belgium & Positive & ‘Low-Low' & 0.05 & Cluster \\
\hline & Austria & Positive & 'Low-Low' & 0.01 & Cluster \\
\hline & France & Positive & 'High-High' & 0.05 & Cluster \\
\hline & Italy & Positive & ‘High-High' & 0.05 & Cluster \\
\hline & Greece & Positive & 'High-High' & 0.01 & Cluster \\
\hline \multirow{6}{*}{ V 2004} & Ireland & Positive & 'High-High' & 0.001 & Cluster \\
\hline & Italy & Positive & 'Low-Low' & 0.001 & Cluster \\
\hline & \begin{tabular}{|l|} 
Belgium \\
\end{tabular} & Negative & 'Low-Low' & 0.05 & Cluster \\
\hline & Netherlands & Negative & 'Low-High' & 0.001 & Outlier \\
\hline & Austria & Negative & ‘Low-High' & 0.001 & Outlier \\
\hline & Germany & Negative & 'Low-High' & 0.001 & Outlier \\
\hline \multirow{5}{*}{ XI 2004} & \begin{tabular}{|l|} 
Portugal \\
\end{tabular} & Positive & 'High-High' & 0.001 & Cluster \\
\hline & Ireland & Positive & ‘High-High' & 0.001 & Cluster \\
\hline & France & Positive & ‘High-High' & 0.001 & Cluster \\
\hline & Greece & Positive & 'High-High' & 0.01 & Cluster \\
\hline & Netherlands & Positive & 'Low-Low' & 0.05 & Cluster \\
\hline \multirow{4}{*}{ V 2005} & \begin{tabular}{|l|} 
Portugal \\
\end{tabular} & Positive & 'High-High' & 0.001 & Cluster \\
\hline & Greece & Positive & 'High-High' & 0.001 & Cluster \\
\hline & Netherlands & Positive & 'Low-Low' & 0.05 & Cluster \\
\hline & France & Negative & ‘High-Low' & 0.05 & Outlier \\
\hline XI 2005 & Netherlands & Positive & 'Low-Low' & 0.05 & Cluster \\
\hline \multirow{6}{*}{ V 2006} & Portugal & Positive & ‘High-High' & 0.001 & Cluster \\
\hline & France & Positive & 'Low-Low' & 0.001 & Cluster \\
\hline & Finland & Positive & 'Low-Low' & 0.05 & Cluster \\
\hline & Germany & Positive & 'Low-Low' & 0.05 & Cluster \\
\hline & Netherlands & Positive & 'Low-Low' & 0.05 & Cluster \\
\hline & Ireland & Negative & 'Low-High' & 0.001 & Outlier \\
\hline \multirow{4}{*}{ XI 2006} & \begin{tabular}{|l|} 
Portugal \\
\end{tabular} & Positive & 'High-High' & 0.05 & Cluster \\
\hline & Spain & Positive & ‘High-High' & 0.001 & Cluster \\
\hline & Greece & Positive & 'High-High' & 0.05 & Cluster \\
\hline & Netherlands & Negative & 'Low-Low' & 0.05 & Cluster \\
\hline \multirow{7}{*}{ V 2007} & Spain & Positive & ‘High-High' & 0.001 & Cluster \\
\hline & Greece & Positive & 'High-High' & 0.001 & Cluster \\
\hline & Slovenia & Positive & 'High-High' & 0.05 & Cluster \\
\hline & Finland & Positive & 'Low-Low' & 0.001 & Cluster \\
\hline & Austria & Positive & 'Low-Low' & 0.001 & Cluster \\
\hline & Netherlands & Positive & 'Low-Low' & 0.001 & Cluster \\
\hline & Belgium & Positive & 'Low-Low' & 0.001 & Cluster \\
\hline \multirow{4}{*}{ XI 2007} & Greece & Positive & 'High-High' & 0.001 & Cluster \\
\hline & Netherlands & Positive & 'Low-Low' & 0.001 & Cluster \\
\hline & Belgium & Positive & 'Low-Low' & 0.001 & Cluster \\
\hline & Finland & Positive & 'Low-Low' & 0.001 & Cluster \\
\hline \multirow{7}{*}{ V 2008} & \begin{tabular}{|l|} 
Spain \\
\end{tabular} & Positive & 'High-High' & 0.001 & Cluster \\
\hline & Finland & Positive & 'Low-Low' & 0.0001 & Cluster \\
\hline & Germany & Positive & ‘Low-Low' & 0.0001 & Cluster \\
\hline & Netherlands & Positive & 'Low-Low' & 0.05 & Cluster \\
\hline & Belgium & Positive & 'Low-Low' & 0.0001 & Cluster \\
\hline & Italy & Positive & 'Low-Low' & 0.0001 & Cluster \\
\hline & Malta & Positive & 'Low-Low' & 0.0001 & Cluster \\
\hline
\end{tabular}




\begin{tabular}{|c|c|c|c|c|c|}
\hline & Austria & Negative & 'High-Low' & 0.0001 & Outlier \\
\hline & Cyprus & Negative & ‘High-Low' & 0.0001 & Outlier \\
\hline & Portugal & Negative & ‘High-Low’ & 0.0001 & Outlier \\
\hline \multirow{8}{*}{ XI 2008} & Spain & Positive & ‘Low-Low' & 0.001 & Cluster \\
\hline & Netherlands & Positive & 'Low-Low' & 0.01 & Cluster \\
\hline & Austria & Negative & ‘Low-High' & 0.001 & Outlier \\
\hline & Italy & Negative & 'Low-High' & 0.001 & Outlier \\
\hline & Portugal & Negative & ‘Low-High' & 0.001 & Outlier \\
\hline & Greece & Negative & ‘High-Low' & 0.001 & Outlier \\
\hline & Cyprus & Negative & ‘High-Low’ & 0.05 & Outlier \\
\hline & Malta & Negative & ‘High-Low' & 0.001 & Outlier \\
\hline \multirow{11}{*}{ V 2009} & Germany & Positive & ‘Low-Low' & 0.001 & Cluster \\
\hline & Austria & Positive & 'Low-Low' & 0.001 & Cluster \\
\hline & Netherlands & Positive & 'Low-Low' & 0.01 & Cluster \\
\hline & Italy & Positive & ‘Low-Low' & 0.001 & Cluster \\
\hline & Slovakia & Positive & ‘Low-Low' & 0.05 & Cluster \\
\hline & France & Positive & 'Low-Low' & 0.001 & Cluster \\
\hline & Finland & Negative & 'Low-High' & 0.001 & Outlier \\
\hline & Belgium & Negative & ‘High-Low' & 0.001 & Outlier \\
\hline & Cyprus & Negative & 'High-Low' & 0.001 & Outlier \\
\hline & Greece & Negative & ‘High-Low' & 0.05 & Outlier \\
\hline & Slovenia & Negative & ‘High-Low' & 0.05 & Outlier \\
\hline \multirow{8}{*}{ XI 2009} & Netherlands & Positive & 'High-High' & 0.001 & Cluster \\
\hline & Slovakia & Positive & ‘High-High' & 0.001 & Cluster \\
\hline & Belgium & Positive & 'Low-Low' & 0.001 & Cluster \\
\hline & Cyprus & Positive & ‘Low-Low' & 0.001 & Cluster \\
\hline & Spain & Positive & ‘Low-Low' & 0.001 & Cluster \\
\hline & Austria & Negative & ‘Low-High' & 0.001 & Outlier \\
\hline & Slovenia & Negative & 'Low-High' & 0.001 & Outlier \\
\hline & Ireland & Negative & ‘High-Low' & 0.001 & Outlier \\
\hline \multirow{11}{*}{ V 2010} & Finland & Positive & ‘High-High' & 0.001 & Cluster \\
\hline & Italy & Positive & ‘High-High' & 0.001 & Cluster \\
\hline & Slovenia & Positive & 'High-High' & 0.001 & Cluster \\
\hline & Cyprus & Positive & ‘Low-Low' & 0.001 & Cluster \\
\hline & Portugal & Positive & ‘Low-Low' & 0.001 & Cluster \\
\hline & Slovakia & Positive & 'Low-Low' & 0.001 & Cluster \\
\hline & Austria & Negative & ‘Low-High' & 0.001 & Outlier \\
\hline & Belgium & Negative & 'Low-High' & 0.001 & Outlier \\
\hline & Germany & Negative & 'Low-High' & 0.001 & Outlier \\
\hline & France & Negative & ‘Low-High' & 0.001 & Outlier \\
\hline & Spain & Negative & ‘High-Low' & 0.001 & Outlier \\
\hline \multirow{4}{*}{ XI 2010} & Slovakia & Positive & 'Low-Low' & 0.001 & Cluster \\
\hline & Spain & Positive & 'Low-Low' & 0.001 & Cluster \\
\hline & Cyprus & Positive & 'High-High' & 0.001 & Cluster \\
\hline & Belgium & Negative & 'High-Low' & 0.001 & Outlier \\
\hline \multirow{2}{*}{ V 2011} & Portugal & Positive & ‘High-High' & 0.05 & Cluster \\
\hline & Netherlands & Positive & ‘Low-Low' & 0.001 & Cluster \\
\hline \multirow{2}{*}{ XI 2011} & Portugal & Positive & 'High-High' & 0.01 & Cluster \\
\hline & Italy & Negative & ‘Low-High' & 0.05 & Outlier \\
\hline \multirow{3}{*}{ V 2012} & Spain & Positive & ‘Low-Low' & 0.05 & Cluster \\
\hline & Slovenia & Positive & 'Low-Low' & 0.05 & Cluster \\
\hline & Cyprus & Positive & ‘Low-High' & 0.05 & Outlier \\
\hline \multirow{2}{*}{ XI 2012} & Belgium & Positive & ‘High-High' & 0.001 & Cluster \\
\hline & Netherlands & Positive & 'High-High' & 0.001 & Cluster \\
\hline
\end{tabular}




\begin{tabular}{|c|c|c|c|c|c|}
\hline & Slovakia & Positive & 'High-High' & 0.001 & Cluster \\
\hline & Finland & Positive & 'Low-Low' & 0.001 & Cluster \\
\hline & Spain & Negative & ‘Low-High' & 0.001 & Outlier \\
\hline & Ireland & Negative & 'Low-High' & 0.001 & Outlier \\
\hline & France & Negative & 'Low-High' & 0.001 & Outlier \\
\hline \multirow{6}{*}{ V 2013} & Austria & Positive & 'High-High' & 0.05 & Cluster \\
\hline & Finland & Positive & 'High-High' & 0.001 & Cluster \\
\hline & Slovenia & Positive & 'High-High' & 0.05 & Cluster \\
\hline & Latvia & Positive & 'Low-Low' & 0.05 & Cluster \\
\hline & Portugal & Positive & 'Low-Low' & 0.01 & Cluster \\
\hline & Spain & Negative & 'Low-High' & 0.05 & Outlier \\
\hline \multirow{6}{*}{ XI 2013} & Finland & Positive & 'High-High' & 0.01 & Cluster \\
\hline & Belgium & Positive & 'High-High' & 0.05 & Cluster \\
\hline & Austria & Positive & 'High-High' & 0.001 & Cluster \\
\hline & Ireland & Positive & 'Low-Low' & 0.05 & Cluster \\
\hline & Latvia & Positive & 'Low-Low' & 0.001 & Cluster \\
\hline & France & Negative & 'Low-High' & 0.05 & Outlier \\
\hline \multirow{5}{*}{ V 2014} & Austria & Positive & 'High-High' & 0.01 & Cluster \\
\hline & Cyprus & Positive & 'Low-Low' & 0.05 & Cluster \\
\hline & Spain & Positive & 'Low-Low' & 0.01 & Cluster \\
\hline & Slovakia & Positive & 'Low-Low' & 0.05 & Cluster \\
\hline & Malta & Negative & ‘Low-High' & 0.05 & Outlier \\
\hline \multirow{7}{*}{ XI 2014} & Austria & Positive & 'High-High' & 0.01 & Cluster \\
\hline & Cyprus & Positive & 'High-High' & 0.01 & Cluster \\
\hline & Spain & Positive & 'Low-Low' & 0.01 & Cluster \\
\hline & Latvia & Negative & 'Low-High' & 0.05 & Outlier \\
\hline & Lithuania & Negative & 'Low-High' & 0.05 & Outlier \\
\hline & Portugal & Negative & 'Low-High' & 0.05 & Outlier \\
\hline & Ireland & Negative & 'High-Low' & 0.01 & Outlier \\
\hline \multirow{5}{*}{ V 2015} & Latvia & Positive & 'High-High' & 0.001 & Cluster \\
\hline & Malta & Positive & 'High-High' & 0.001 & Cluster \\
\hline & Spain & Positive & 'Low-Low' & 0.05 & Cluster \\
\hline & Portugal & Negative & 'Low-High' & 0.01 & Outlier \\
\hline & Cyprus & Negative & 'Low-High' & 0.05 & Outlier \\
\hline \multirow{4}{*}{ XI 2015} & France & Positive & 'High-High' & 0.05 & Cluster \\
\hline & Germany & Negative & 'High-Low' & 0.001 & Outlier \\
\hline & Estonia & Negative & 'High-Low' & 0.001 & Outlier \\
\hline & Latvia & Negative & 'High-Low' & 0.01 & Outlier \\
\hline \multirow{7}{*}{ V 2016} & Austria & Positive & 'High-High' & 0.01 & Cluster \\
\hline & Malta & Positive & 'High-High' & 0.05 & Cluster \\
\hline & Latvia & Negative & 'Low-High' & 0.05 & Cluster \\
\hline & Germany & Negative & 'High-Low' & 0.001 & Cluster \\
\hline & Finland & Negative & 'High-Low' & 0.001 & Outlier \\
\hline & Italy & Negative & 'High-Low' & 0.001 & Outlier \\
\hline & France & Negative & 'High-Low' & 0.001 & Outlier \\
\hline \multirow{2}{*}{ XI 2016} & Spain & Positive & 'Low-Low' & 0.01 & Cluster \\
\hline & Italy & Negative & 'High-Low' & 0.05 & Outlier \\
\hline
\end{tabular}

Significant at ${ }^{*} \mathrm{p}<0.1,{ }^{* *} \mathrm{p}<0.01,{ }^{* * *} \mathrm{p}<0.001$

Note: the sample changes due to the new Member States' accession to the euro area.

Source: own calculations.

Regardless of the number of local correlations detected by the Local Moran's test, we can find no pattern of local dependence. The old euro area Member States are 
clusters/outliers in approximately $10-15$ cases out of 30 , but on some occasions a Member State is the cluster's centre and on others it is an outlier. Neither Germany - the largest EU economy - nor any countries currently experiencing severe economic disturbances, such as Greece, Spain, or Portugal, seem to be permanent clusters/outliers. We have not found any pattern that would make it possible to track down the countries that are more prone to clustering than are others. The results depend neither on the ECB's effectiveness in achieving inflation goals nor on the national inflation rates and their volatility over time. None of the eleven economies proved to be a clear "information hub" with a tendency to accumulate expectations around it. Even if economic deployment seems more important than is geographic deployment, it is still quite random: we see no room for drawing conclusions that substantiate impact on policies. Other than the existence of some clusters, we have not discerned any cross-country homogeneity of expectations that undermines our initial assumption of their heterogeneity.

Economic deployment that does not reveal any pattern on either the global or the local level is rather bad news for policy-makers. Even similar inflation rates are not enough to ensure correlations of consumer expectations among the Member States. The same nominal interest rate would mean different real interest rates for consumers. Their decisions that drives savings and consumption would bring different results in terms of economic performance. The same real interest rate does not guarantee the same monetary transmission, but it facilitates it to some extent. A similar conclusion on the geographic deployment of expectations can be found in Gnan, Langthaler, \& Valderrama (2011): the results of a cross-country examination of expectations demonstrated that high geographical heterogeneities exist. The signs and values of coefficients in the regression analysis varied significantly.

\section{Conclusion}

In this paper, we examined the spatial deployment of consumers' expectations among euro area Member States. We applied a novel methodology: the spatial approach. The results suggest that spatial deployment of consumers' expectations expressed by economic relations among the Member States is not homogenous. Thus, we confirmed the results presented in previous research with the use of the methodology appropriate to examination of spatial deployment. With the use of the economic distance weights (inflation rates), we found some global correlation of the results, but we did not find any Member States that cluster permanently the other countries with similar inflation rates.

Given the lack of general coordination of expectations expressed in time-varying global correlations and no clustering rule, national factors and national information are of substantial importance. The explanation stems from economic conditions, demography, or culture. The disparities of the patterns of formation of national expectations make implementation of the monetary policy demanding. However, expectations' heterogeneity 
could also offer an opportunity of easier accommodation in comparison to the situation when homogenous expectations are wrongly anchored, especially at too low a level.

We recognise that this examination needs to be expanded, including a search for alternative weighting of economic distance and coverage of the year-to-year analysis to present the results. We are aware that a more elaborate way of detecting the spatial dependence exists: i.e., spatial regression analysis. We did not apply it because our sample was enlarged during the research period. Moreover, as this research is pioneering, we decided to start from more simple measures of spatial deployment to check whether there is room for further examination.

The most important conclusion from this research could refer to the critique of Anselin (1988) that we mentioned in the introduction: random geographic deployment of expectations in the euro area legitimates the application of traditional methodologies.

\section{References}

Anselin, L. \& Florax R.J.G.M. (1995a). Small sample properties of tests for Spatial Dependence in Regression Models: Some further results. In New directions in spatial econometrics. Berlin: Springer, 21-74.

Anselin, L. (1988). Spatial econometrics: Methods and models. Dordrecht: Kluwer Academic Publishers.

Anselin, L. (1995). Local indicators of spatial associations-LISA. Geographical Analysis. 27(2), 93-115. doi:10.1111/j.1538-4632.1995.tb00338.x.

Anselin, L. (2005). Exploring spatial data with GeoDaTM: A workbook, Centre for Spatially Integrated Social Science, derived from: http://sal.uiuc.edu/.

Anselin, L. Florax, R. \& Rey, S. (2004). Advances in spatial econometrics. Methodology, tools and applications. Berlin: Springer. doi:10.1007/978-3-662-05617-2.

Batchelor, R.A., \& Orr, A. B. (1988). Inflation expectations revisited. Economica, New Series. 55(219), 317331. doi:10.2307/2554010.

Berger, H., Ehrmann, M. \& Fratzscher, M. (2009). Forecasting ECB monetary policy: accuracy is a matter of geography. European Economic Review. 58(8), 1028-1041. doi:10.1016/j.euroecorev.2008.12.003.

Berger, H., Ehrmann, M. \& Fratzscher, M. (2011). Geography, skills or both: What explains Fed watchers‘ forecasts accuracy of US monetary policy? Journal of Macroeconomics. 33, 420-437. doi:10.1016/j.jmacro.2011.03.002.

Carlson, J.A., \& Parkin, J. M. (1975). Inflation expectations. Economica. 42, 123-138. doi:10.2307/2553588.

Cerisola, M. \& Gelos, G. (2009). What drives inflation expectations in Brazil? An empirical analysis. Applied Economics. 41(10), 1215-1227. doi:10.1080/00036840601166892.

Ciccarelli, M. \& Garcia, J. A. (2009). What drives euro area break even inflation rates? ECB Working Paper Series, 996, Frankfurt am Mein: European Central Bank.

Cliff, A.D. \& Ord, J.K. (1981). Spatial processes: models and applications. London: Pion.

Cruijsen van der, C., \& Strobach, Ch. (2015). The formation of European inflation expectations: One learning rule does not fit all. De Nederlandsche Bank Working Paper. 472. 
Gambetti, L. \& Moretti, L. (2016). What drives inflation expectations? from: https://editorialexpress.com/cgibin/conference/download.cgi?db_name=EEAESEM2016\&paper_id=2 290

Gimeno, R. \& Ortega, E. (2016). The evolution of inflation expectations in euro area markets. Documentos de Trabajo Banco de Espana. 1627.

Gnan, E., Langthaler, J. \& Valderrama, M. (2011). Heterogeneity in euro area consumers' inflation expectations: Some stylized facts and implications. Monetary Policy and the Economy. Q2 June. Oesterreichische Nationalbank, 43-66.

Tobler, R. W. (1970). A computer movie simulating urban growth in the Detroit region. Economic Geography. 46 (2), 234-240. doi:10.2307/143141.

Weber, A. (2009). Heterogeneous expectations, learning and European inflation dynamics, from:https://www.newyorkfed.org/medialibrary/media/research/conference/2010/consumer/Paper_A Weber.pdf. 